Conflict of interest: A. Ercan is the founder of UtopicPharma LLC. M. Pezer is an employee of the Genos Clycoscience Research Laboratory. E.W. Yu holds an investigator-initiated grant from Seres Therapeutics Inc. G. Lauc is the founder and chief executive officer of Genos and holds several glycobiology patents (PK20130568A, US20160103137, CB2361699). K.D. Deane holds investigator-initiated grants from Pfizer Inc. and Janssen Pharmaceuticals Inc. U.B. Kaiser has equity in Merck, GlycoMimetics, ImmunoCen, Blueprint Medicines, Agios, Express Scripts, Akebia, Amgen, Celgene, Hologic, Johnson \& Johnson, Pfizer Inc., and Teva as well as spousal income from ImmunoGen, GlycoMimetics, Blueprint Medicines, Idera, aTyr, and Imara. J.S. Finkelstein holds an investigator-initiated grant from AbbVie Inc. P.A. Nigrovic receives salary support as a member of the steering committee of the Childhood Arthritis and Rheumatology Research Alliance; authorship royalties from UpToDate Inc. and the American Academy of Pediatrics; and holds investigator-initiated grants from Novartis Inc. and Sobi Inc.

Submitted: July 21, 2016 Accepted: January 10, 2017 Published: February 23, 2017

Reference information: JCI Insight. 2017;2(4):e89703. https:// doi.org/10.1172/jci.insight.89703.

\section{Estrogens regulate glycosylation of IgG in women and men}

\author{
Altan Ercan, ${ }^{1,2}$ Wendy M. Kohrt, ${ }^{3}$ Jing Cui, ${ }^{1}$ Kevin D. Deane, ${ }^{4}$ Marija Pezer, ${ }^{5}$ Elaine W. Yu, ${ }^{6}$ \\ Jonathan S. Hausmann, ${ }^{7,8}$ Harry Campbell, ${ }^{9}$ Ursula B. Kaiser, ${ }^{10}$ Pauline M. Rudd, ${ }^{11}$ Cordan Lauc, ${ }^{5,12}$ \\ James F. Wilson, ${ }^{9,13}$ Joel S. Finkelstein, ${ }^{6}$ and Peter A. Nigrovic ${ }^{1,7}$ \\ 'Division of Rheumatology, Immunology and Allergy, Brigham and Women's Hospital, Boston, Massachusetts, USA. \\ ${ }^{2}$ UtopicPharma LLC, Odessa, Florida, USA. ${ }^{3}$ Division of Ceriatrics and ${ }^{4}$ Division of Rheumatology, University of Colorado \\ Denver School of Medicine, Aurora, Colorado, USA. ${ }^{5}$ Cenos Glycoscience Research Laboratory, Zagreb, Croatia. ${ }^{6}$ Endocrine \\ Unit, Department of Medicine, Massachusetts General Hospital, Boston, Massachusetts, USA. 'Division of Immunology, \\ Boston Children's Hospital, Boston, Massachusetts, USA. ${ }^{8}$ Division of Rheumatology, Beth Israel Deaconess Medical \\ Center, Boston, Massachusetts, USA. ${ }^{9}$ Centre for Global Health Research, Usher Institute for Population Health Sciences \\ and Informatics, University of Edinburgh, Edinburgh, Scotland. ${ }^{10}$ Division of Endocrinology, Diabetes and Hypertension, \\ Brigham and Women's Hospital, Boston, Massachusetts, USA. "National Institute for Bioprocessing Research and \\ Training, University College Dublin, Dublin, Ireland. ${ }^{12}$ Faculty of Pharmacy and Biochemistry, University of Zagreb, \\ Zagreb, Croatia. ${ }^{13} \mathrm{MRC}$ Human Genetics Unit, Institute of Genetics and Molecular Medicine, University of Edinburgh, \\ Western General Hospital, Edinburgh, Scotland.
}

The immunologic potency of IgC is modulated by glycosylation, but mechanisms regulating this process are undefined. A role for sex hormones is suggested by differences in IgC glycans between women and men, most prominently with respect to galactose. We therefore assessed IgC galactosylation in 713 healthy adults from 2 cohorts as well as in 159 subjects from 4 randomized controlled studies of endocrine manipulation: postmenopausal women receiving conjugated estrogens, raloxifene, or placebo; premenopausal women deprived of gonadal hormones with leuprolide and treated with estradiol or placebo; men deprived of gonadal hormones with goserelin and given testosterone or placebo; and men deprived of gonadal hormones with goserelin and given testosterone or placebo together with anastrozole to block conversion of testosterone to estradiol. Menopause was associated with an increase in agalactosylated IgC glycans, particularly in the most abundant fucosylated nonbisected (GOF) glycoform. Conjugated estrogens and raloxifene reduced GOF glycans in postmenopausal women, while in premenopausal women leuprolide increased GOF glycans in a manner reversed by estradiol. Among men, goserelin increased GOF glycans, an effect blocked by testosterone through conversion to estradiol. These results establish estrogens as an in vivo modulator of IgC galactosylation in both women and men, defining a pathway by which sex modulates immunity.

\section{Introduction}

The capacity of IgG to mediate immune responses is determined in part by two glycans within the Fc region that modulate binding to $\mathrm{Fc}$ receptors and complement (1). Each glycan can assume over 30 different forms (Figure 1), a diversity that affords the opportunity to fine-tune humoral immunity. For example, in patients with HIV, a shift toward proinflammatory IgG glycans correlates with more effective antiviral defense, while in rheumatoid arthritis glycan changes may contribute to the ability of IgG to trigger pathogenic inflammation (2-6). Glycoforms lacking terminal galactose (termed G0, denoting zero galactoses, see Figure 1) are particularly proinflammatory because they confer an enhanced ability to fix complement and to engage the activating IgG receptor Fc $\gamma$ RIIIa while simultaneously blocking antiinflammatory mechanisms mediated through sialylated and/or bigalactosylated (G2) glycans (2, 4, 7, 8).

Despite the immunological importance of IgG glycosylation, its regulation is poorly understood. Epidemiological data suggest that endocrine factors may play a role. Population studies reveal an increase in G0 glycans in midlife in women but not in men (9-12). During pregnancy, a marked 
$\mathrm{N}$-acetylglucosamine

Fucose

Mannose

Galactose

Sialic acid

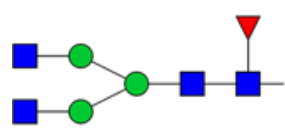

G0F

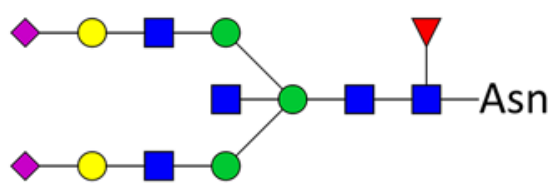

Figure 1. Schematic representation of IgG Fc glycans. The Fc region of IgG contains two glycans, one per heavy chain, attached to the single conserved glycosylation site at asparagine (Asn) 297. The most complex glycan, presented at the top, contains 13 monosaccharide units, including 2 galactoses (i.e., C2), a bisecting $N$-acetylglucosamine, a core fucose attached to core $\mathrm{N}$-acetylglucosamine, and terminal sialic acids. Other IgG Fc glycans are similar in overall structure but lack one or more monosaccharide. These smaller forms include the three presented below: GOF (no galactoses, no bisecting $N$-acetylglucosamine, with core fucose), GOFB (same as GOF, with a bisecting $\mathrm{N}$-acetylglucosamine), and G1F (galactose attached to either the $\alpha 1,6$ or $\alpha 1,3$ arm).

decline in G0 fraction accompanies the second and third trimesters, which reverses within 3 months of parturition $(13,14)$. Estradiol, the primary circulating form of estrogen, and to a lesser extent progesterone exhibit correlations between spot hormone levels and IgG glycans, particularly with respect to differential galactosylation (12). Thus, estrogens and potentially other hormones are plausible candidate modulators of IgG glycosylation, though the observational nature of available data leaves a direct etiologic connection uncertain.

Interestingly, estrogen production is not limited to women. While the male testis elaborates only small amounts of estrogens directly, estradiol is routinely generated from testosterone through the action of aromatase (also termed estrogen synthase) (15). In men, estradiol participates in skeletal maturation, body fat regulation, and sexual function (16). Thus, an effect of estrogens on IgG glycosylation could be physiologically relevant in men as well as women. Whether testosterone directly alters IgG glycans has not been explored.

The consequences of a role for sex hormones in the determination of IgG glycosylation are potentially substantial. Levels of these hormones not only differentiate men and women but also vary widely within an individual across the life span. Further, related medical interventions are common, including postmenopausal replacement therapy, oral contraception, testosterone supplementation, and aromatase inhibition. We therefore sought to test the effect of estrogens and testosterone on human IgG galactosylation in vivo.

\section{Results}

G0 IgG increases in association with menopause. We used liquid chromatography to assess IgG glycans in 126 male and 119 female adult blood donors, employing monogalactosylated (G1) glycans as a normalizing factor (17). G0/G1 glycans increased with age (Figure 2A). Dividing G0 glycoforms into their two major subclasses, fucosylated nonbisected G0 (G0F) and fucosylated bisected G0 (G0FB), we found that the magnitude of age-related change was most prominent in the more abundant G0F component (Figure $2 B$ ). G0F/G1 glycans increased with age in both women and men ( $\beta$ per year of age $=0.003 \pm 0.002, P$ $=0.038$ vs. $\beta=0.008 \pm 0.002, P<0.0001)$. Below 50 years of age, G0F/G1 glycans were significantly lower in women than men $(P<0.0001$, adjusted for age), a difference no longer evident in older individuals. Correspondingly, employing $\geq 50$ years of age as an interaction term in linear regression, we found a prominent increase in $\mathrm{G} 0 \mathrm{~F} / \mathrm{G} 1$ glycans in women only, consistent with a potential role for menopause ( $\beta$ $=0.16 \pm 0.05, P=0.004$; men $\beta=-0.07 \pm 0.05, P=\mathrm{NS}$ ) (Figure $2 \mathrm{C}$ ). Neither women nor men showed an age group-dependent increment in G0FB/G1 (both $\beta=0.00 \pm 0.03, P=\mathrm{NS}$ ) (Figure 2C).

We confirmed this observation in a second cohort of 468 adult donors recruited from the Orkney Islands, Scotland, employing purified IgG to quantitate glycans without G1 normalization (18). Again, below 50 years of age, women exhibited a lower proportion of G0F IgG glycans than men $(P<0.001$, corrected for age); this difference was no longer observed at $\geq 50$ years. Whereas G0F increased gradually with age in both women and men $(\beta=0.22 \pm 0.04, P<0.0001$ vs. $\beta=0.26 \pm 0.04, P<0.0001)$, only women exhibited an increase in G0F glycans at age $50(\beta=4.46 \pm 1.16 P=0.0002$; men $\beta=-0.41$ $\pm 1.30 P=$ NS) (Figure 2D and Supplemental Figure 1; supplemental material available online with this article; https://doi.org/10.1172/jci.insight.89703DS1). This higher-resolution method identified a parallel increase in G0FB glycans, again for women only, although with a much smaller magnitude of shift (Supplemental Figure 1). 

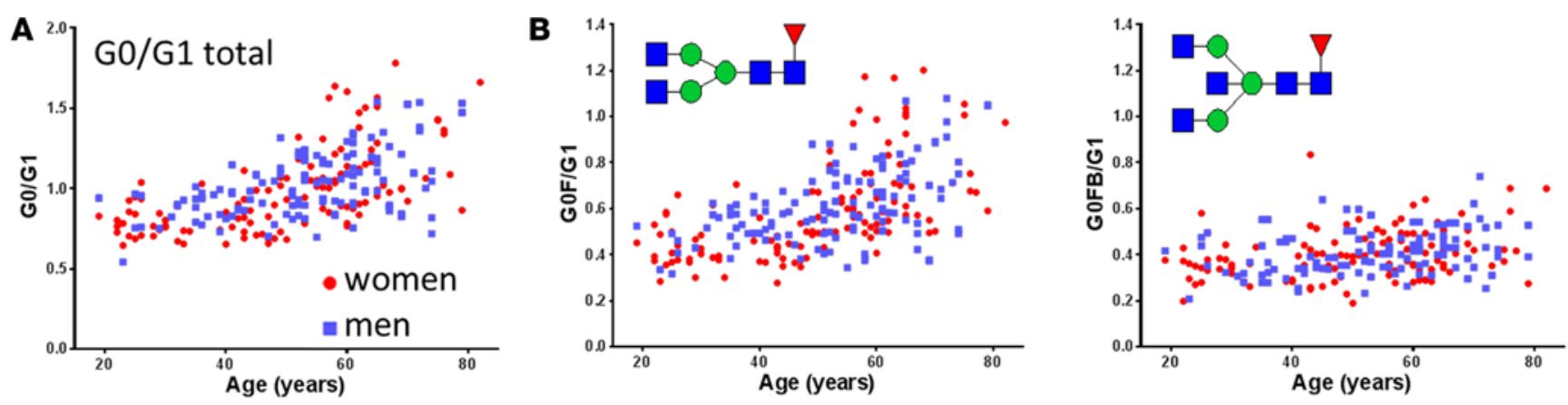

C
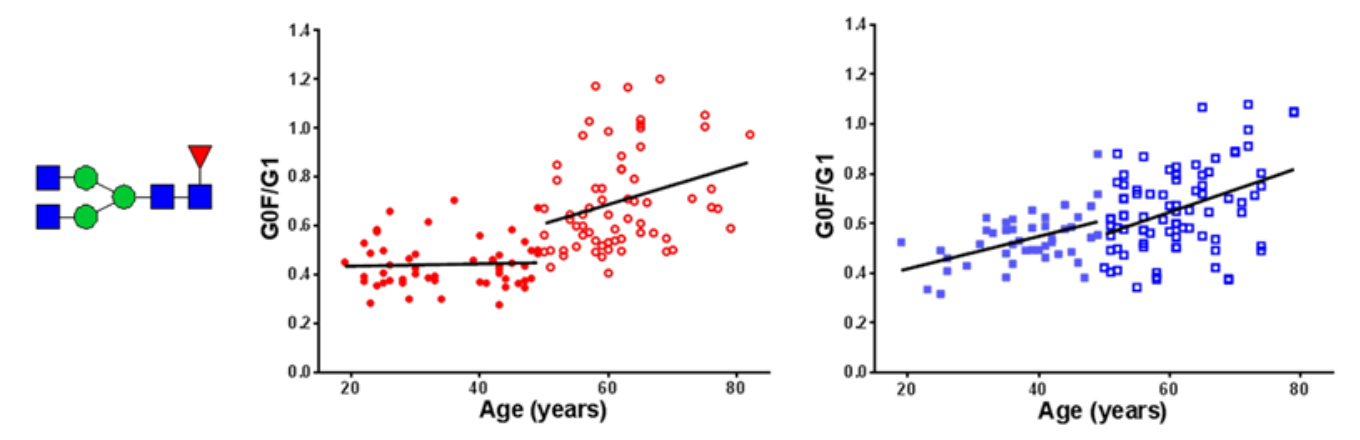

- women $<50$ y
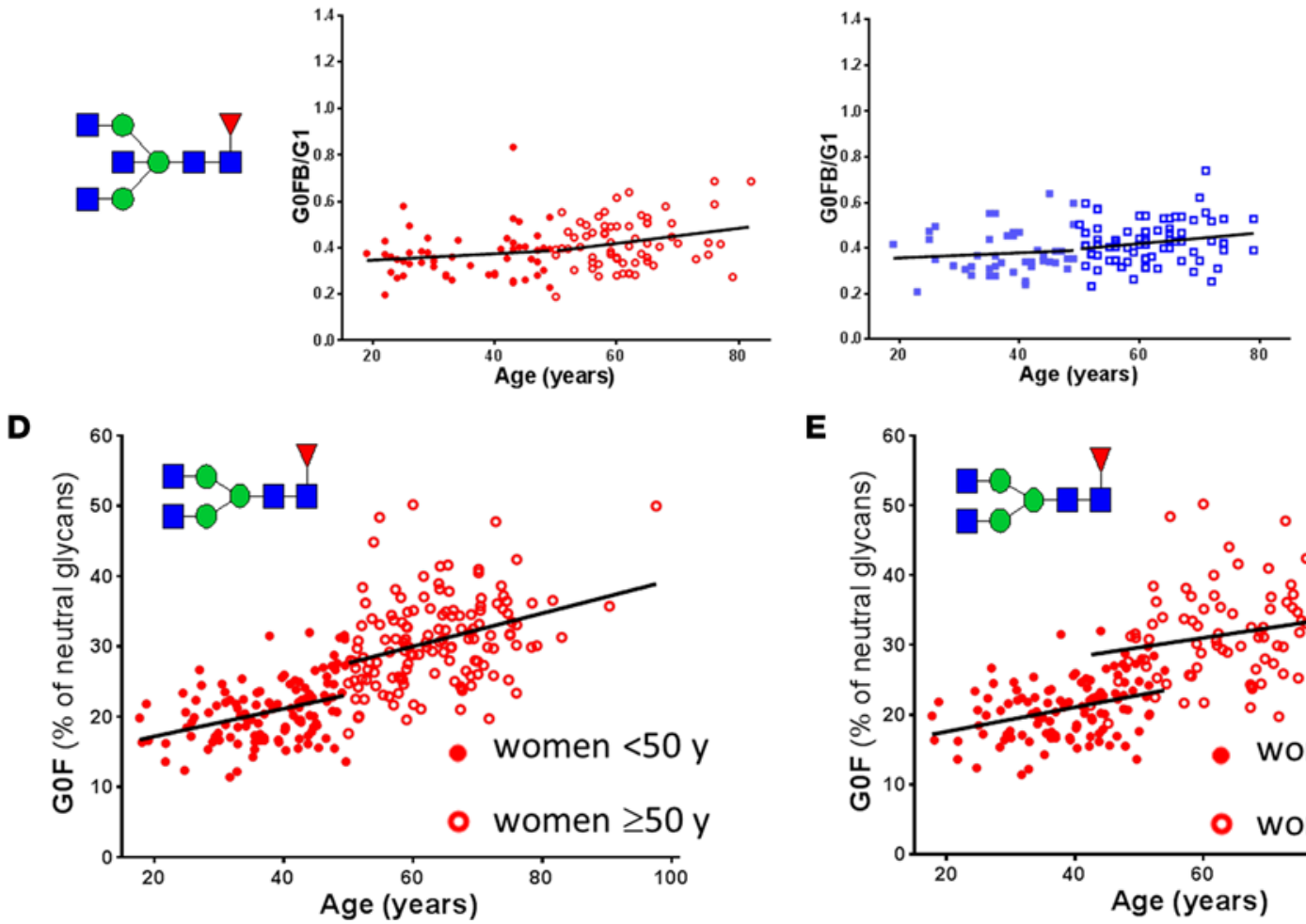

$\mathbf{E}$

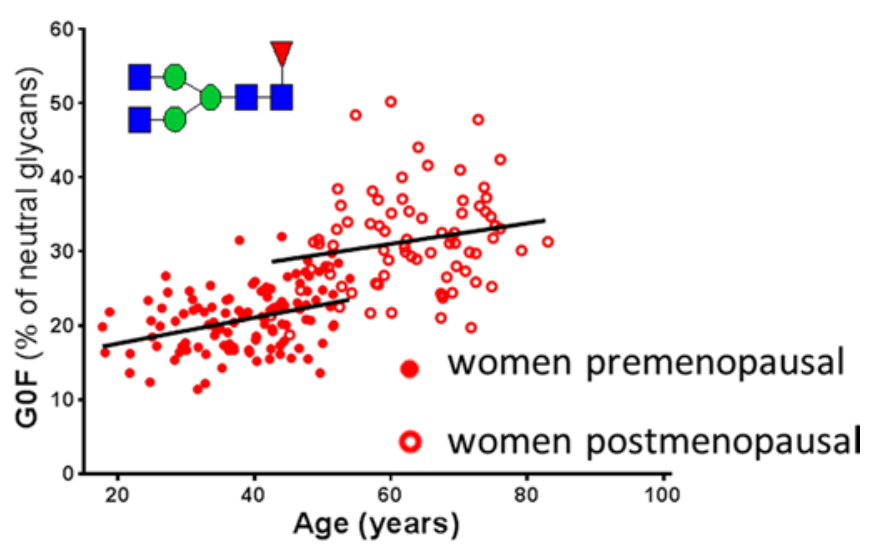

Figure 2. Agalactosylated IgG glycoforms increase in association with menopause. (A) Serum glycans were quantitated by HPLC in a cohort of 245 blood donors (119 women, 126 men). G0/G1 glycans increased with age (Pearson $r=0.58, P<0.0001$ ). (B) Normalized to G1 glycans, both G0F and G0FB glycans increased with age (GOF/G1 $r=0.57, P<0.0001$; GOFB/G1 $r=0.30, P<0.0001$ ). (C) Divided at 50 years of age, women exhibited an increase in G0F/G1 glycans; no similar increase was noted in men. Neither women nor men showed an age group-dependent increase in GOFB/G1 glycans. (D) IgG-specific glycans quantitated by UPLC in 261 women from the Orkney cohort demonstrated an increase in GOF glycans at 50 years of age. (E) IgC galactosylation in 189 women from the Orkney cohort, as per self-declared menopausal status, excluding postmenopausal women receiving hormone supplementation.

Taking advantage of clinical data within the Orkney cohort, we compared premenopausal women ( $n$ $=112$ ) with women who reported cessation of menses and who had never received hormone supplementation $(n=77)$. This correction strengthened the G0F increment ( $\beta$ for menopause $=6.74 \pm 1.30, P<0.0001$, compared with $\beta$ per year of age $=0.16 \pm 0.04, P<0.0001$ ) (Figure $2 \mathrm{E}$ ). Interestingly, in premenopausal 

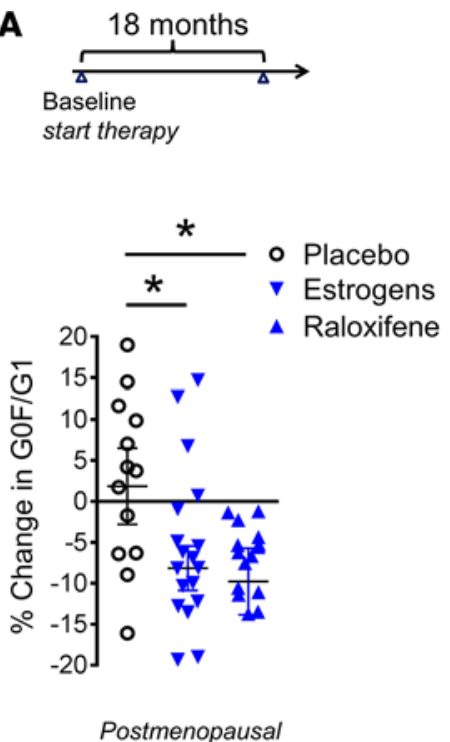

B
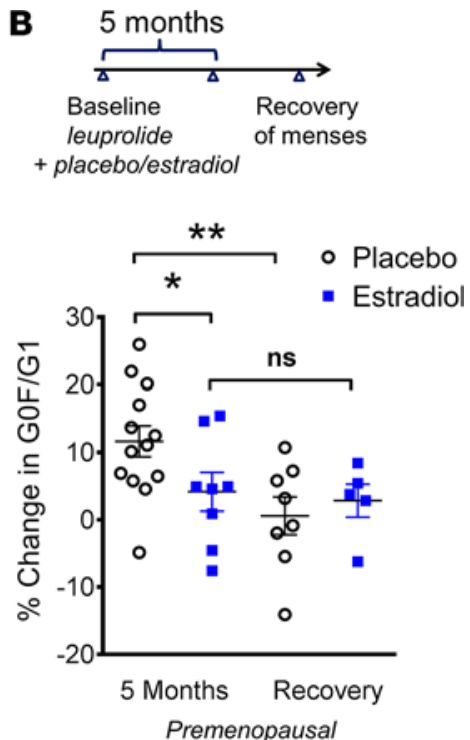

Figure 3. Estrogen receptor agonists modulate IgG glycosylation in vivo. (A) Postmenopausal women were treated orally with placebo, conjugated estrogens, or raloxifene daily, and serum G0/G1 glycans were assessed after 18 months ( $n=18-20$ ) group). (B) Premenopausal women were treated with leuprolide to induce gonadal quiescence and supplemented with placebo or estradiol. GO/G1 glycans were assessed 5 months after start of therapy and at recovery of menses ( $n=5-13$ /group). ${ }^{*} P<0.05$, ${ }^{* *} P<0.01$ by 2 -tailed $t$ test using unpaired statistics.

women for whom information was available concerning oral contraceptive use, self-reported current utilization ( $n$ $=29)$ compared with no oral contraceptive $(n=81)$ was associated with a modest reduction in G0F glycans corrected for age $(\beta=-1.88 \pm 0.91, P=0.04)$.

Estrogen agonists reduce GOF glycans in postmenopausal and premenopausal women. To evaluate the specific contribution of gonadal hormones, we obtained blood samples from 58 postmenopausal women randomized to placebo, conjugated estrogens ( $0.625 \mathrm{mg}$ daily), or raloxifene (60

mg daily), regimens employed for alleviation of postmenopausal vasomotor symptoms and/or osteoporosis (19). Both conjugated estrogens and raloxifene reduced G0F/G1 glycans compared with placebo $(P<0.05)$ (Figure 3A). We then assessed IgG glycans in 21 healthy premenopausal women treated on an investigational basis with the gonadotropin-releasing hormone $(\mathrm{GnRH})$ analog leuprolide to lower gonadal steroids to postmenopausal levels and then randomized to placebo or transdermal estradiol $(0.075 \mathrm{mg} / \mathrm{d})$ (20). G0F/G1 glycans increased in placebo recipients compared with estrogen recipients $(P=0.03$, Figure $3 \mathrm{~B})$, while the cohorts became indistinguishable once the GnRH agonist effect faded sufficiently for normal menses to resume (Figure 3B). These results confirm that estrogens drive IgG galactosylation and implicate estrogen deficiency in the increase in G0F glycans that accompanies menopause.

Estrogens derived from testosterone promote Ig G galactosylation in men. Compared with premenopausal women, men exhibit lower levels of circulating estrogens, principally as estradiol derived from testosterone by aromatase (21). To evaluate whether sex steroids alter IgG glycans in men, we tested samples from healthy male volunteers treated with the GnRH agonist goserelin, as employed for testosterone deprivation therapy in prostate cancer, followed by placebo or $5 \mathrm{~g}$ of testosterone gel daily (16). In placebo recipients, serum testosterone and estradiol levels remained in the prepubertal range, while testosterone restored both hormones to the midportion of the adult reference range (Figure 4A). Intriguingly, G0F/G1 glycans rose in placebo recipients but remained stable in testosterone recipients (Figure 4A). To evaluate whether this effect reflected testosterone itself or rather testosterone-derived estradiol, we tested samples from men receiving the same interventions in the presence of the aromatase inhibitor anastrozole (1 mg daily), a treatment that resulted in levels of estradiol remaining equally low in testosterone and placebo groups (Figure 4B). Under these conditions, testosterone no longer normalized G0F/G1 glycans, confirming that its effect on IgG galactosylation was mediated not directly by testosterone itself but instead by testosterone-derived estradiol (Figure 4B).

\section{Discussion}

The relationship between sex and immune function is complex. Not all immunological differences between men and women are hormonal. For example, genes of immune relevance are found on the sex chromosomes (22). However, multiple immune lineages, including B cells, express estrogen and androgen receptors, implicating gonadal steroids in immune regulation (23). Understanding the role of hormones in immune function is important because these effects will give rise to immune differences between men and women as well as in association with childhood, puberty, pregnancy, and menopause. Further, an effect of sex hormones on immune function could lead to unintended consequences of the therapeutic manipulation of gonadal steroids.

In this study, we show that estrogens modulate human IgG galactosylation in vivo. Measurable changes in IgG glycans occur during menopause and from administration of hormones at therapeutic doses, including postmenopausal hormone replacement, oral contraception, and testosterone supple- 
A

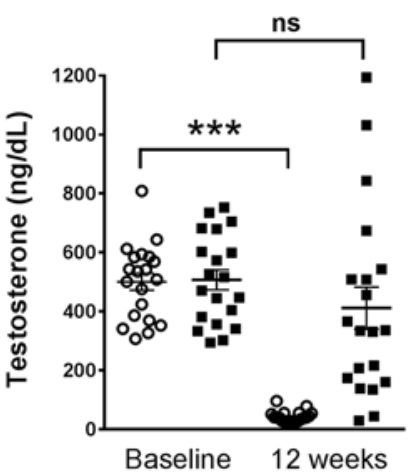

B

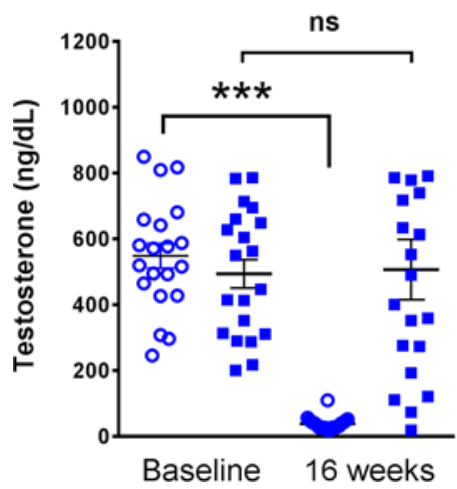

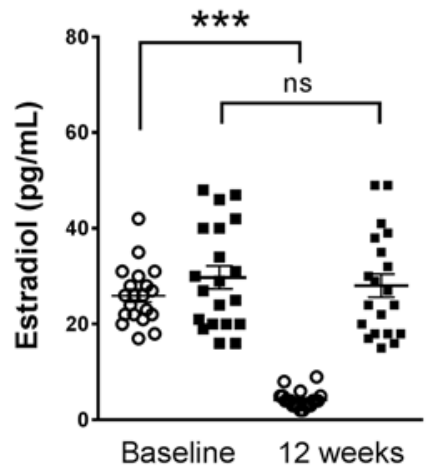

O goserelin + placebo

goserelin + testosterone

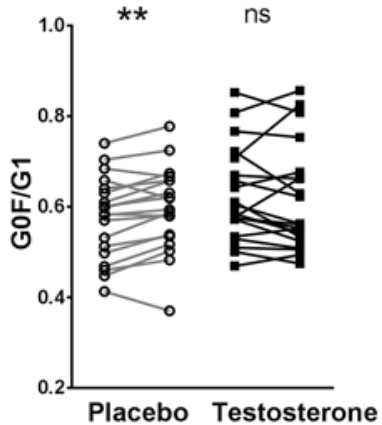

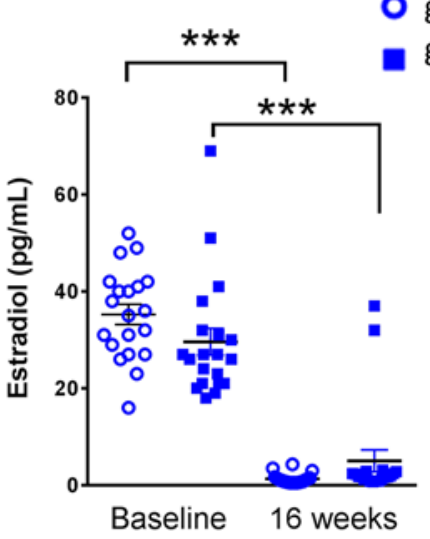

goserelin/anastrozole + placebo goserelin/anastrozole + testosterone

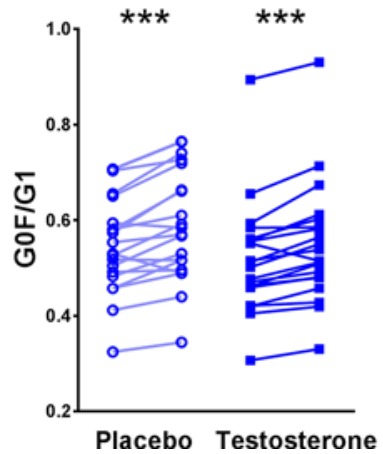

Figure 4. Estrogens regulate IgG glycosylation in men. (A) Serum testosterone and estradiol levels at baseline and 12 weeks in men treated with goserelin acetate and placebo or testosterone and changes in GOF/G1 glycans in response to these hormonal manipulations ( $n=20 /$ group). (B) Serum testosterone and estradiol levels at baseline and 16 weeks in men treated with goserelin acetate plus anastrozole together with placebo or testosterone and changes in GOF/G1 glycans in response to these hormonal manipulations ( $n=20$ /group). ${ }^{* *} P<0.01,{ }^{* *} P<0.001$, paired 2-tailed $t$ test. The leftmost panel excludes from display (but not from analysis or error bars) an outlier in the 16-week placebo group (testosterone 1,858 $\mathrm{ng} / \mathrm{dl}$ ).

mentation. This mechanism is operative in men as well, reflecting the action of estradiol derived from aromatization of testosterone rather than testosterone itself. These observations identify a pathway by which sex, as well as related medical interventions, affect immunity.

Altered IgG galactosylation is of particular interest because of the pivotal role of galactose on IgG effector function. G0 glycans enable IgG to engage mannose binding lectin, enhancing complement fixation via the lectin pathway $(4,24)$. G0 glycans also enhance the affinity of IgG for Fc $\gamma$ RIIIIa and therefore promote antibody-dependent cellular cytotoxicity, an effect implicated in antiviral potency (2). Whereas G1 glycans remain relatively stable across the adult age range, the increase in G0 glycans translates into a reduction in G2 glycans that engage the inhibitory IgG receptor Fc $\gamma$ RIIb and dectin- 1 to block chemoattractant signaling (8, 25). Moreover, only galactosylated Fc glycans are able to bear terminal sialic acid (see Figure 1), a modification that impairs $F_{C}$ receptor binding while engaging a range of antiinflammatory mechanisms $(7,26-28)$. These considerations suggest that estrogens are likely to constrain the inflammatory capacity of the IgG pool.

One evolutionarily critical milieu in which the effect of estrogen on IgG could play a role is pregnancy. Pregnant women demonstrate a marked decrease in IgG G0 glycans $(13,14)$. Estradiol levels increase as much as 100 -fold by the third trimester of pregnancy, such that the mechanism identified here is likely to be an important driver of the pregnancy-associated decline in agalactosylated IgG (29). Complement fixation is implicated in fetal demise, raising the possibility that estrogen-mediated glycan changes promote successful gestation (30).

Hormonal effects on IgG glycans could also affect immune-mediated disease. Autoantibody-containing IgG immune complexes and complement fixation are implicated in diseases such as rheumatoid arthritis (31). Rheumatoid arthritis is strongly associated with an increase in G0 IgG glycans, even preceding disease 
onset $(3,32,33)$. Further, the extent of glycan aberrancy correlates with disease severity (34). In pregnancy, reduction in G0 glycans correlates with improvement of rheumatoid arthritis disease activity, reversing in the postpartum period at a time of high risk for disease resurgence (13). Elevation in G0 glycans also occurs in vasculitis, juvenile idiopathic arthritis, systemic lupus erythematosus, and inflammatory bowel disease as well as during normal aging $(3,11,32,35-38)$. Recent studies have directly implicated cytokine-driven changes in IgG glycosylation (in particular sialylation) in the ability of murine IgG to induce experimental arthritis, corresponding to lower levels of sialylation and galactosylation in both total and autoantigen-specific IgG1 among asymptomatic human subjects who proceeded to develop rheumatoid arthritis within 12 months $(5,6)$. The effect of estrogens on this disease biology remains to be determined. Despite the female predominance of rheumatoid arthritis, the effect of estrogens observed here would ordinarily be expected to render antibodies less inflammatory. Consistent with this possibility, the incidence of rheumatoid arthritis among women rises in the immediate postmenopausal years, and premature menopause is associated with higher disease risk. Postmenopausal hormone supplementation appears to afford minimal, if any, protection perhaps because IgG glycosylation represents only one of many factors associated with disease risk (39-43).

We recognize important limitations to our study. The data herein do not address the mechanism by which estrogens alter IgG glycans. Although ligation of estrogen receptors on B cells is an appealing candidate, estrogen receptors are widely distributed, and it is possible that the effect of estrogens on glycosylation of IgG is fully or partially mediated by the effect of other lineages on B cell maturation and function. We have not determined whether the effect of estrogens on galactosylation extends to proteins beyond IgG. Indeed, a more widespread phenomenon is suggested by a cross-sectional study of plasma glycans that identified a general increase in G0 forms in women over the age of 50 years compared with younger women, potentially broadening the physiologic effect of estrogen-driven galactosylation (44).

A further limitation stems from our methodology. Most of our results were obtained using HPLC to characterize glycans from whole serum or plasma. In this context, relative specificity of G0 and G1 glycoforms for IgG arises because most N-linked glycans outside of the sterically restricted IgG Fc region are more highly developed (i.e., G2 and larger) (17). Even within the G0 subfraction, IgG depletion studies show that, while the large majority of G0F glycans originate with IgG, a substantial fraction of the minor G0FB component arises from other circulating proteins (data not shown). This effect likely explains why our HPLC studies found an estrogen-related difference only in G0F, while a statistically significant but much smaller menopause-associated shift in G0FB glycans could be detected in the Orkney analysis of purified IgG (Supplemental Figure 1). We therefore suspect that the effect of estrogens also extends to the lower-frequency (and even more proinflammatory) G0FB glycoform, although the utilization of HPLC for our experimental samples rendered us unable to test this possibility directly.

For the same reason, our methodology allowed us to address only IgG galactosylation, despite the importance of features such as fucosylation and sialylation to IgG function. Gender differences have been noted to extend to these features as well $(9,12)$. Galactosylation is required for attachment of sialic acid (see Figure 1), and galactosylation and sialylation frequently exhibit marked colinearity $(2,12)$. It is therefore possible that, by providing more galactose substrate, estrogens could indirectly promote greater IgG Fc sialylation. Consistent with this possibility, women but not men manifest a decrease in IgG Fc sialylation around the transition between the fifth and sixth decade of life (9). Further study is required to assess whether gonadal hormones affect sialylation, fucosylation, and other glycan features independently of galactosylation.

In summary, the abundance of G0 IgG glycans increases in women in association with menopause. Studies in healthy women undergoing endocrine manipulation indicate that this shift is mediated, at least in part, by the ability of estrogen to promote IgG galactosylation. Estradiol derived from aromatization of testosterone exerts a similar effect on IgG glycans in men. Taken together, these findings confirm that estrogens regulate human IgG glycosylation in vivo and thereby establish a mechanism through which sex modulates immunity.

\section{Methods}

Study participants. We assessed IgG glycosylation patterns in 6 cohorts. (a) The first cohort consisted of 245 blood donors from Brigham and Women's Hospital, with samples obtained as discarded samples characterized only by age and gender. (b) The second cohort consisted of 468 consented adult volunteer participants in the Orkney Complex Disease Study (ORCADES), Orkney Islands $(18,32)$. (c) The third cohort consisted of 58 healthy postmenopausal women (age $56 \pm 4$ years, age at menopause $48 \pm 6$ years) randomized to oral therapy with placebo, conjugated estrogens (Premarin, Pfizer Inc.) (0.625 mg daily), or raloxifene 
(Evista, Eli Lily Inc.) (60 mg daily) for 18 months, selected at random from a larger study $(n=119)$ of the metabolic effects of hormone replacement (19). Blood samples were collected at baseline and at the end of the intervention. Women randomized to conjugated estrogens whose uteri were intact also received medroxyprogesterone acetate ( $5 \mathrm{mg}$ daily) for 13 consecutive days trimonthly for endometrial protection; placebo and raloxifene subjects received cyclic placebo. (d) The fourth cohort consisted of 21 healthy premenopausal women (age $34 \pm 7$ years) treated with 5 monthly intramuscular doses of the GnRH analog leuprolide acetate (TAP Pharmaceutical Products Inc.) $(3.75 \mathrm{mg}$ ) to suppress endogenous gonadal steroids and then randomized to concurrent treatment with transdermal estradiol (Bayer HealthCare Pharmaceuticals Inc.) $(0.075 \mathrm{mg} / \mathrm{d})$ or placebo. Patients were selected at random from a larger study $(n=70)$ of the metabolic effects of estradiol in premenopausal women (20). (e) The fifth cohort consisted of 40 healthy male volunteers (age $33 \pm 7$ years) treated with the $\mathrm{GnRH}$ agonist goserelin acetate (Zoladex, AstraZeneca Inc.) (3.6 mg subcutaneously at weeks $0,4,8$, and 12) to suppress endogenous gonadal steroids and then randomized to simultaneous administration of placebo or testosterone gel (AndroGel, AbbVie Inc.) $5 \mathrm{~g}$ daily. Samples were analyzed for glycans at baseline and at 12 weeks. (f) The last cohort consisted of 40 healthy male volunteers (age $34 \pm 6$ years) treated with goserelin acetate (Zoladex, AstraZeneca Inc.) (3.6 mg subcutaneously at weeks $0,4,8$, and 12) plus anastrozole (Arimidex, AstraZeneca Inc.) (1 mg orally daily) to block aromatization of testosterone to estradiol and then randomized to simultaneous administration of placebo or testosterone gel (5 g daily). Samples were analyzed for glycans at baseline and at 16 weeks. Cohorts e and $\mathrm{f}$ were selected at random from subjects in a larger study in which men were randomized to a range of doses of testosterone gel (placebo, 1.25, 2.5, 5, or $10 \mathrm{~g}$ daily), with $(n=198)$ or without $(n=202)$ administration of anastrozole; the $5 \mathrm{~g}$ testosterone group was selected as the comparator for this post-hoc study, because this dose maintained levels of gonadal steroids similar to baseline (16). Participants in all studies were unaware of study group assignment. Descriptions of the various cohorts, the interventions, and study results have been published $(16,19,20,45)$.

IgG glycan analysis. Samples from blood donors and hormonally manipulated subjects were analyzed using an established method $(17,32)$. Glycans were cleaved enzymatically from $5 \mu$ of serum or plasma, conjugated to the fluorochrome 2-aminobenzamide, and quantitated using a TSKgel Amide-80 5- $\mu \mathrm{m}$ column attached to a Waters 1525 binary HPLC pump with a Waters 2487 dual wavelength detector, a Waters 2475 multifluorescence detector, and a Waters 717plus autosampler. To permit comparison across individuals, G0 glycans were normalized to the G1 fraction, which is stable across the adult age range $(17,25)$. In this method, selectivity for IgG glycans arises because the large majority of G0 and G1 forms in serum or plasma are from the IgG Fc region (17). Samples from ORCADES donors were analyzed from IgG purified by plate-bound protein G, using ultra performance liquid chromatography (UPLC), as described previously (18). For both methods, analysis was restricted to neutral glycans in order to avoid contamination from glycans attached to the Fab region of some IgG, since these are predominantly sialylated. HPLC studies were performed at the Brigham and Women's Hospital, and UPLC studies were performed at the Genos Glycoscience Research Laboratory; within each comparison, samples were collected and analyzed simultaneously to avoid batch effects. The specific glycan fractions quantitated under HPLC and UPLC protocols are depicted in Supplemental Figure 2 and Supplemental Figure 3, respectively.

Statistics. Variance in glycoform abundance with age was assessed using Pearson statistics. Comparison of galactosylation in men and women, adjusted for age, employed general linear regression. An interaction term for age group ( $<50$ vs. $\geq 50$ years), menopause (yes/no by self-report), or oral contraceptive use (by self-report) was incorporated to assess associated increments in galactosylation ( $\beta$ coefficient \pm SEM). Comparison among experimentally manipulated subjects was performed using Student's $t$ tests ( 2 tailed) for data normally distributed per Shapiro-Wilks and by Mann-Whitney $U$ tests for nonparametric data. Error bars represent SEM. Statistical tests were performed using SAS 9.4 or GraphPad Prism 6 software. Significance was established at $\alpha=0.05$.

Study approval. The analyses reported here represent secondary use of previously collected samples, conducted under Brigham and Women's Hospital institutional review board approval P2004000060.

\section{Author contributions}

AE and PAN conceptualized and designed the research. AE, PMR, and GL acquired data. AE, JC, and PAN analyzed data. WK, KDD, MP, EWY, JSH, HC, UBK, JFW, and JSF provided samples, reagents, and other project assistance. PAN wrote the manuscript, and all authors edited the manuscript. 


\section{Acknowledgments}

This work was supported by the NIH Office of Research on Women's Health, National Institute of Child Health and Human Development, Building Interdisciplinary Research Careers in Women's Health grant K12 HD051959 (to AE and UBK); the Arthritis National Research Foundation (to AE); NIH grants R01 AG018198 and P50 HD073063 (to WK), P30 AR070253 (to JC), R01 HD019938 and HD082314 (to UBK), and R21 AI099435, R03 AR061602, and P30 AR070253 (to PAN); and the Rheumatology Research Foundation, the Cogan Family Foundation, and the Fundación Bechara (to PAN). ORCADES was supported by the Chief Scientist Office of the Scottish government, the Royal Society, the MRC Human Genetics Unit, Arthritis Research UK, and the European Union framework program 6 EUROSPAN project (contract LSHG-CT-2006-018947). Studies in men given goserelin acetate and testosterone gel with or without anastrozole were supported by NIH grants R01 AG 030545, K24 DK0275, and S10 RR02305 (to JSF) and M01 RR01066 and UL1 RR 25758 (to the Massachusetts General Hospital Clinical Research Center). AbbVie Inc. provided product and funding for the investigator-initiated study "Dose Response of Gonadal Steroids and Bone Turnover in Men." Lab values and samples from that study were used as the basis for the data on men in this publication. The authors thank Wendolyn Gozansky for assistance in obtaining samples for this project.

Address correspondence to: Peter A. Nigrovic, Brigham and Women's Hospital, Building for Transformative Medicine 6002L, 60 Fenwood Road, Boston, Massachusetts 02115, USA. Phone 617.525.1031; E-mail: pnigrovic@partners.org.

1. Lux A, Nimmerjahn F. Impact of differential glycosylation on IgG activity. Adv Exp Med Biol. 2011;780:113-124.

2. Ackerman ME, et al. Natural variation in Fc glycosylation of HIV-specific antibodies impacts antiviral activity. J Clin Invest. 2013;123(5):2183-2192.

3. Parekh RB, et al. Association of rheumatoid arthritis and primary osteoarthritis with changes in the glycosylation pattern of total serum IgG. Nature. 1985;316(6027):452-457.

4. Malhotra R, Wormald MR, Rudd PM, Fischer PB, Dwek RA, Sim RB. Glycosylation changes of IgG associated with rheumatoid arthritis can activate complement via the mannose-binding protein. Nat Med. 1995;1(3):237-243.

5. Ohmi Y, et al. Sialylation converts arthritogenic IgG into inhibitors of collagen-induced arthritis. Nat Commun. 2016;7:11205.

6. Pfeifle R, et al. Regulation of autoantibody activity by the IL-23-TH17 axis determines the onset of autoimmune disease. Nat Immunol. 2017;18(1):104-113.

7. Kaneko Y, Nimmerjahn F, Ravetch JV. Anti-inflammatory activity of immunoglobulin G resulting from Fc sialylation. Science 2006;313(5787):670-673.

8. Karsten CM, et al. Anti-inflammatory activity of IgG1 mediated by Fc galactosylation and association of Fc $\gamma$ RIIB and dectin-1. Nat Med. 2012;18(9):1401-1406.

9. Baković MP, et al. High-throughput IgG Fc N-glycosylation profiling by mass spectrometry of glycopeptides. J Proteome Res. 2013;12(2):821-831.

10. Ding N, et al. Human serum N-glycan profiles are age and sex dependent. Age Ageing. 2011;40(5):568-575.

11. Krištić J, et al. Glycans are a novel biomarker of chronological and biological ages. J Gerontol A Biol Sci Med Sci. 2014;69(7):779-789.

12. Chen G, et al. Human IgG Fc-glycosylation profiling reveals associations with age, sex, female sex hormones and thyroid cancer. J Proteomics. 2012;75(10):2824-2834.

13. van de Geijn FE, et al. Immunoglobulin G galactosylation and sialylation are associated with pregnancy-induced improvement of rheumatoid arthritis and the postpartum flare: results from a large prospective cohort study. Arthritis Res Ther. 2009;11(6):R193.

14. Ruhaak LR, Uh HW, Deelder AM, Dolhain RE, Wuhrer M. Total plasma N-glycome changes during pregnancy. J Proteome Res. 2014;13(3):1657-1668.

15. Longcope C, Kato T, Horton R. Conversion of blood androgens to estrogens in normal adult men and women. J Clin Invest. 1969;48(12):2191-2201.

16. Finkelstein JS, et al. Gonadal steroids and body composition, strength, and sexual function in men. N Engl J Med. 2013;369(11):1011-1022.

17. Royle L, et al. HPLC-based analysis of serum N-glycans on a 96-well plate platform with dedicated database software. Anal Biochem. 2008;376(1):1-12.

18. Pucić M, et al. High throughput isolation and glycosylation analysis of IgG-variability and heritability of the IgG glycome in three isolated human populations. Mol Cell Proteomics. 2011;10(10):M111.010090.

19. Van Pelt RE, et al. Estrogen or raloxifene during postmenopausal weight loss: adiposity and cardiometabolic outcomes. Obesity (Silver Spring). 2014;22(4):1024-1031.

20. Shea KL, et al. Body composition and bone mineral density after ovarian hormone suppression with or without estradiol treatment. Menopause. 2015;22(10):1045-1052.

21. Simpson ER. Sources of estrogen and their importance. J Steroid Biochem Mol Biol. 2003;86(3-5):225-230.

22. Libert C, Dejager L, Pinheiro I. The X chromosome in immune functions: when a chromosome makes the difference. Nat Rev Immunol. 2010;10(8):594-604. 
23. Hughes GC, Choubey D. Modulation of autoimmune rheumatic diseases by oestrogen and progesterone. Nat Rev Rheumatol. 2014;10(12):740-751.

24. Arnold JN, Dwek RA, Rudd PM, Sim RB. Mannan binding lectin and its interaction with immunoglobulins in health and in disease. Immunol Lett. 2006;106(2):103-110.

25. Parekh R, Roitt I, Isenberg D, Dwek R, Rademacher T. Age-related galactosylation of the N-linked oligosaccharides of human serum IgG. JExp Med. 1988;167(5):1731-1736.

26. Anthony RM, Wermeling F, Karlsson MC, Ravetch JV. Identification of a receptor required for the anti-inflammatory activity of IVIG. Proc Natl Acad Sci USA. 2008;105(50):19571-19578.

27. Anthony RM, Kobayashi T, Wermeling F, Ravetch JV. Intravenous gammaglobulin suppresses inflammation through a novel $\mathrm{T}(\mathrm{H}) 2$ pathway. Nature. 2011;475(7354):110-113.

28. Pincetic A, et al. Type I and type II Fc receptors regulate innate and adaptive immunity. Nat Immunol. 2014;15(8):707-716.

29. Tulchinsky D, Hobel CJ, Yeager E, Marshall JR. Plasma estrone, estradiol, estriol, progesterone, and 17-hydroxyprogesterone in human pregnancy. I. Normal pregnancy. Am J Obstet Gynecol. 1972;112(8):1095-1100.

30. Lynch AM, Salmon JE. Dysregulated complement activation as a common pathway of injury in preeclampsia and other preg nancy complications. Placenta. 2010;31(7):561-567.

31. Firestein GS. Evolving concepts of rheumatoid arthritis. Nature. 2003;423(6937):356-361.

32. Ercan A, et al. Aberrant IgG galactosylation precedes disease onset, correlates with disease activity, and is prevalent in autoantibodies in rheumatoid arthritis. Arthritis Rheum. 2010;62(8):2239-2248.

33. Rombouts Y, et al. Anti-citrullinated protein antibodies acquire a pro-inflammatory Fc glycosylation phenotype prior to the onset of rheumatoid arthritis. Ann Rheum Dis. 2015;74(1):234-241.

34. Ercan A, et al. Hypogalactosylation of serum N-glycans fails to predict clinical response to methotrexate and TNF inhibition in rheumatoid arthritis. Arthritis Res Ther. 2012;14(2):R43.

35. Holland M, et al. Hypogalactosylation of serum IgG in patients with ANCA-associated systemic vasculitis. Clin Exp Immunol. 2002;129(1):183-190.

36. Trbojević Akmačić I, et al. Inflammatory bowel disease associates with proinflammatory potential of the immunoglobulin G glycome. Inflamm Bowel Dis. 2015;21(6):1237-1247.

37. Vuckovic F, et al. Association of systemic lupus erythematosus with decreased immunosuppressive potential of the IgG glycome. Arthritis Rheumatol. 2015;67(11):2978-2989.

38. Ercan A, et al. Multiple juvenile idiopathic arthritis subtypes demonstrate proinflammatory IgG glycosylation. Arthritis Rheum. 2012;64(9):3025-3033.

39. Humphreys JH, Verstappen SM, Hyrich KL, Chipping JR, Marshall T, Symmons DP. The incidence of rheumatoid arthritis in the UK: comparisons using the 2010 ACR/EULAR classification criteria and the 1987 ACR classification criteria. Results from the Norfolk Arthritis Register. Ann Rheum Dis. 2013;72(8):1315-1320.

40. Wong LE, et al. Effect of age at menopause on disease presentation in early rheumatoid arthritis: results from the Canadian Early Arthritis Cohort. Arthritis Care Res (Hoboken). 2015;67(5):616-623.

41. Walitt B, et al. Effects of postmenopausal hormone therapy on rheumatoid arthritis: the women's health initiative randomized controlled trials. Arthritis Rheum. 2008;59(3):302-310.

42. Orellana C, Saevarsdottir S, Klareskog L, Karlson EW, Alfredsson L, Bengtsson C. Postmenopausal hormone therapy and the risk of rheumatoid arthritis: results from the Swedish EIRA population-based case-control study. Eur J Epidemiol. 2015;30(5):449-457.

43. Salliot C, Bombardier C, Saraux A, Combe B, Dougados M. Hormonal replacement therapy may reduce the risk for RA in women with early arthritis who carry HLA-DRB $1{ }^{*} 01$ and/or ${ }^{*} 04$ alleles by protecting against the production of anti-CCP: results from the ESPOIR cohort. Ann Rheum Dis. 2010;69(9):1683-1686.

44. Knezevic A, et al. Effects of aging, body mass index, plasma lipid profiles, and smoking on human plasma N-glycans. Glycobiology. 2010;20(8):959-969.

45. Melanson EL, et al. Regulation of energy expenditure by estradiol in premenopausal women. $J$ Appl Physiol. 2015;119(9):975-981. 